Tendências em Matemática Aplicada e Computacional, 3, No. 1 (2002), 1-14.

(C) Uma Publicação da Sociedade Brasileira de Matemática Aplicada e Computacional.

\title{
Collisions and Fracture of Solids
}

M. FRÉMOND, Laboratoire Lagrange, LCPC, 58 boulevard Lefebvre, 75732 Paris, France.

\begin{abstract}
When a rock collides with a concrete wall, both the rock and the wall can be damaged even split into pieces. We address this problem involving damage theory and collision theory.
\end{abstract}

\section{Introduction}

Consider a rock falling on a concrete wall. The collision can damage both the rock and the wall. They can even be split into pieces: this is the fracture phenomenon.

There are collision theories and damage theories which are based on basic laws of mechanic. We recall them briefly before using them to describe the collision of the rock with the concrete wall.

The collision theories [1], [3], [4], [6] consider that the duration of the collision is very short and assume that the velocities are discontinuous functions of time. They introduce a new term in the work of the interior forces which is related to the discontinuity of the velocities. These theories apply to collisions of either rigid or deformable bodies, even to collisions of solids and fluids.

The damage theories [2], [4], [8] quantify the damage by a quantity $\beta(\vec{x}, t)$ with value 1 when the material is undamaged, value 0 when it is completely damaged and values between 0 and 1 when it is partially damaged. The function $\beta(\vec{x}, t)$ satisfies the constraint

$$
0 \leq \beta(\vec{x}, t) \leq 1 .
$$

The damage theories take into account the microscopic motions which are responsible for the damage evolution.

We use the ideas of these theories to build a predictive theory for collisions producing damage. The equations of motion in a damaging collision are derived from the principle of virtual work where new interior forces are introduce to describe the very large stresses and the very large contact forces resulting from the cinematic incompatibilities. The rules to get the constitutive laws are established with the two laws of thermodynamics. Examples of constitutive laws are given and it is shown that they agree with the basic experimental facts, for instance the incoming velocities have to be large enough to damage partially or to fracture the solids. In all what follows we neglect the thermal effects. 


\section{The damage theory}

Damage, for instance damage of concrete, results from microscopic motions which break the links which ensure the cohesion of the material. The basic idea of the theory is that the power of these microscopic motions must be accounted for in a predictive theory. Thus we decide that the power of the interior forces depends on the damage rate $d \beta / d t$ which is clearly related to the microscopic motions. Furthermore we assume that it depends also on the gradient of the damage rate $\overrightarrow{\operatorname{grad}}(d \beta / d t)$ to account for microscopic interactions.

\subsection{Virtual Power of the Interior Forces}

The virtual power of the interior forces to a subdomain $\mathcal{D}$ of the domain $\Omega$ occupied at time $t$ by the structure under consideration is a linear function of the virtual strain rates $D(\vec{V})=\left((1 / 2)\left(V_{i, j}+V_{j, i}\right)\right)(\vec{V}$ is a virtual velocity) and the virtual damage velocities $b$ and $\overrightarrow{g r a d} b$ which satisfies the axiom of the virtual power of the interior forces:

Axiom 1. At any time the virtual power of the interior forces $\mathcal{P}_{i}(\mathcal{D}, \vec{V}, b)$ to a subdomain $\mathcal{D}$ of the domain $\Omega$ occupied by the structure, is zero for any rigid body velocity.

The rigid body velocities are such that the distance of material points remains constant. Thus no microscopic motion occurs in such a motion. It results that the rigid body velocities are such that $D(\vec{V})=0$, and $b=0, \overrightarrow{g r a d} b=0$. We choose as virtual power of the interior forces

$$
\forall(\mathcal{D}, \vec{V}, b), \mathcal{P}_{i}(\mathcal{D}, \vec{V}, b)=-\int_{\mathcal{D}} \sigma: D(\vec{V}) d \Omega-\int_{\mathcal{D}}\{B b+\vec{H} \cdot \overrightarrow{\operatorname{grad}} b\} d \Omega
$$

The first term on the right-hand is classical. Note that it cannot represent the power of interior forces resulting from microscopic motions which can occur without macroscopic motion. The second term on the right-hand side is unusual. It represents the power of interior forces related to the microscopic motions. The quantity $B$ is a volume density of energy. The vector $\vec{H}$ is an energy flux vector. The equation of motion which is to be found, will give physical meaning to these quantities. Note that the situation is the same with the stress tensor $\sigma$, the physical meaning of which is given by an equation of motion. It will be seen that $\vec{H}$, like $\sigma$, describes the effects of the neighbourhood of a point on this point.

It is clear that the chosen power of interior forces $\mathcal{P}_{i}(\mathcal{D}, \vec{V}, b)$ satisfies the axiom of the virtual power of interior forces.

\subsection{Virtual Power of the Exterior Forces}

The virtual power of exterior forces to a subdomain $\mathcal{D}$ of $\Omega$ is the sum of the power

of the actions at a distance and of the power of the contact actions. Each of them 
is a linear function of $\vec{V}$ and $b$. The virtual power of the actions at a distance is defined by

$$
\int_{\mathcal{D}} \vec{f} \cdot \vec{V} d \Omega+\int_{\mathcal{D}} A b d \Omega
$$

The first term is classical, the second is not. The quantity $A$ is a volume density of energy supplied to the material from the exterior by microscopic actions without macroscopic motion. For instance, $A$ can be the energy supplied by irradiation or by an electrical or chemical action which modifies the microscopic bonds (it is known that radiations damage metals). The energy $A$ can also describe the energy supplied by microscopic mechanical actions which are not taken into account by macroscopic strain rates, for instance, very fast vibrations with vanishing amplitude [5].

The virtual power of contact actions is defined by

$$
\int_{\partial \mathcal{D}} \vec{T} \cdot \vec{V} d \Gamma+\int_{\partial \mathcal{D}} a b d \Gamma
$$

where $\partial \mathcal{D}$ is the boundary of the domain $\mathcal{D}$. Again the first term is classical and, the second is not. The quantity a represents the surface density of energy supplied by the exterior of $\mathcal{D}$ to $\mathcal{D}$ without any macroscopic motion.

Thus the virtual power of exterior forces to the subdomain $\mathcal{D}$ is defined by

$$
\forall(\mathcal{D}, \vec{V}, b), \mathcal{P}_{e}(\mathcal{D}, \vec{V}, b)=\int_{\mathcal{D}} \vec{f} \cdot \vec{V} d \Omega+\int_{\mathcal{D}} A b d \Omega+\int_{\partial \mathcal{D}} \vec{T} \cdot \vec{V} d \Gamma+\int_{\partial \mathcal{D}} a b d \Gamma
$$

\subsection{Virtual Power of the Acceleration Forces}

The virtual power of the acceleration forces is defined by the classical formula

$$
\forall(\mathcal{D}, \vec{V}, b), \mathcal{P}_{a}(\mathcal{D}, \vec{V}, b)=\int_{\mathcal{D}} \rho \vec{\gamma} \cdot \vec{V} d \Omega,
$$

where $\vec{\gamma}=d \vec{U} / d t$ is the acceleration ( $\vec{U}$ is the actual velocity) and $\rho$ the density of the material.

Remark 1. It is possible to take into account the acceleration forces of the microscopic motions. It is wise to define their virtual power by

$$
\int_{\mathcal{D}} \hat{\rho} \hat{\gamma} b d \Omega
$$

where $\hat{\gamma}$ is the material derivative of $d \beta / d t$. The quantity $\hat{\rho}$ is proportional to the mass of the bonds [8].

\subsection{The Equations of Motion}

The principle of virtual power is

$$
\forall(\mathcal{D}, \vec{V}, b), \mathcal{P}_{a}(\mathcal{D}, \vec{V}, b)=\mathcal{P}_{i}(\mathcal{D}, \vec{V}, b)+\mathcal{P}_{e}(\mathcal{D}, \vec{V}, b) .
$$


By letting $b=0$ in (2.1), we obtain the classical equations for the macroscopic motion

$$
\begin{gathered}
\rho \vec{\gamma}=\operatorname{div} \sigma+\vec{f}, \text { in } \mathcal{D}, \\
\sigma \vec{N}=\vec{T}, \text { in } \partial \mathcal{D},
\end{gathered}
$$

where $\vec{N}$ is the exterior outward normal vector to $\mathcal{D}$. By letting $\vec{V}=0$ in (2.1), an easy computation gives the new equations for the microscopic motions

$$
\begin{gathered}
0=\operatorname{div} \vec{H}-B+A, \text { in } \mathcal{D}, \\
\vec{H} \cdot \vec{N}=a, \text { in } \partial \mathcal{D} .
\end{gathered}
$$

The relationship (2.5) gives the physical meaning of $\vec{H}: \vec{H}$ is the energy flux vector and $\vec{H} \cdot \vec{N}$ is the surface density of energy supplied to $\mathcal{D}$ by surface microscopic actions due to the exterior of $\mathcal{D}$ and without macroscopic motion.

\subsection{The constitutive laws}

They are defined by the free energy $\Psi$ and a pseudopotential of dissipation $\Phi$ as introduced by J.J. Moreau [7], i.e., a positive convex function with value 0 at the origin. We choose

$$
\Psi(\epsilon, \beta, \overrightarrow{\operatorname{grad}} \beta)=\frac{1}{2} \beta \epsilon: K: \epsilon+w(1-\beta)+\frac{k}{2}(\overrightarrow{\operatorname{grad}} \beta)^{2}+I(\beta),
$$

where $I$ is the indicator function of the segment $[0,1]$ taking into account the constraint (1.1), (see appendix), $K$ is the elasticity tensor, $\epsilon=\epsilon(\vec{u})$ is the small deformation ( $\vec{u}$ is the small displacement), and $w$ is the cohesion energy of the material. The positive parameter $k$ measures the influence of the microscopic bonds onto their immediate neighbourhood;

$$
\Phi\left(\frac{d \beta}{d t}\right)=\frac{c}{2}\left(\frac{d \beta}{d t}\right)^{2}+I_{-}\left(\frac{d \beta}{d t}\right),
$$

where $c$ is a viscosity parameter, $I_{-}$is the indicator function of the set of the negative numbers (see appendix). The indicator function implies that

$$
\frac{d \beta}{d t} \leq 0
$$

or that the material when damaged does not mend by itself. The constitutive laws are

$$
\begin{gathered}
\sigma=\frac{\partial \Psi}{\partial \epsilon}=\beta K: \epsilon \\
B=\frac{\partial \Psi}{\partial \beta}+\frac{\partial \Phi}{\partial(d \beta / d t)} \in \frac{1}{2} \epsilon: K: \epsilon-w+c \frac{d \beta}{d t}+\partial I(\beta)+\partial I_{-}\left(\frac{d \beta}{d t}\right), \\
\vec{H}=\frac{\partial \Psi}{\partial \overrightarrow{\operatorname{grad} \beta}}=k \overrightarrow{g r a d} \beta .
\end{gathered}
$$




\subsection{The equations}

The equations of motion (2.2)-(2.5) and the constitutive laws (2.6)-(2.8) give the set of partial differential equations which describe the evolution of a dammageable structure. We assume that the evolution is quasi-static $(\vec{\gamma}=0)$ and that no energy is provided by the exterior to the structure: $A=0$ and $a=0$. The structure is fixed on $\Gamma$ to a fixed rigid support. The equations are

$$
\begin{gathered}
\operatorname{div}(\beta K: \epsilon(\vec{u}))+\vec{f}=0, \text { in } \Omega, \vec{u}=0 \text { in } \Gamma, \sigma \vec{N}=0, \text { in } \partial \Omega / \Gamma, \\
c \frac{d \beta}{d t}-k \Delta \beta+\partial I(\beta)+\partial I_{-}\left(\frac{d \beta}{d t}\right) \ni w-\frac{1}{2} \epsilon(\vec{u}): K: \epsilon(\vec{u}), \text { in } \Omega, \\
\frac{\partial \beta}{\partial N}=0, \text { in } \partial \Omega .
\end{gathered}
$$

completed by an initial condition for $\beta$. The unknowns of this set of partial differential equations are the small displacement $\vec{u}$ and the damage $\beta$. These equations are discussed in [8].

\section{The collision theory}

\subsection{The equations of motion}

Let us consider a deformable solid colliding a rigid fixed plane at time $t$ on contact surface $\Gamma(t)$. Due to the cinematic incompatibilities very large interior forces appear within the solid $\Omega(t)$ and on contact surface $\Gamma(t)$. We consider the duration of the collision is very short, i.e., the time for the velocities to adapt to the presence of the obstacle is very short. Thus we assume the collision is instantaneous: the velocity field before the collision is denoted $\vec{U}^{-}(\vec{x}, t)$ and the velocity field after the collision is denoted $\vec{U}^{+}(\vec{x}, t)$. The very large interior forces become percussion stresses $\Sigma(\vec{x}, t)$ and percussions $\vec{R}(\vec{x}, t)$ Their virtual work in the collision is

$$
\mathcal{T}_{i}(\vec{V})=-\int_{\Omega(t)} \Sigma: D\left(\frac{\vec{V}^{+}+\vec{V}^{-}}{2}\right) d \Omega-\int_{\Gamma(t)} \vec{R} \cdot\left(\frac{\vec{V}^{+}+\vec{V}^{-}}{2}\right) d \Gamma
$$

where the virtual velocity field $\vec{V}(\vec{x}, \tau)$ is a function of bounded variation (thus it may be discontinuous at time $t$ ). The virtual work of the acceleration forces is

$$
\mathcal{T}_{a}(\vec{V})=\int_{\Omega(t)} \rho[\vec{U}] \cdot \frac{\vec{V}^{+}+\vec{V}^{-}}{2} d \Omega
$$

where $\rho$ is the density and $[\vec{U}]=\vec{U}^{+}-\vec{U}^{-}$is the velocity discontinuity. For the sake of completeness, we assume an external percussion $\vec{T}_{\text {ext }}$ concomitant to the collision may be applied on the boundary of the solid $\partial \Omega(t) / \Gamma(t)$

$$
\mathcal{T}_{e}(\vec{V})=\int_{\partial \Omega(t) / \Gamma(t)} \vec{T}_{e x t} \cdot \frac{\vec{V}^{+}+\vec{V}^{-}}{2} d \Gamma .
$$


The equations of motion in the collision result from the principle of virtual work

$$
\forall \vec{V}, \mathcal{T}_{a}(\vec{V})=\mathcal{T}_{i}(\vec{V})+\mathcal{T}_{e}(\vec{V})
$$

They are

$$
\begin{gathered}
\rho[\vec{U}]=\operatorname{div} \Sigma, \text { in } \Omega(t), \\
\Sigma \vec{N}=-\vec{R}, \text { in } \Gamma(t), \Sigma \vec{N}=\vec{T}_{e x t}, \text { in } \partial \Omega(t) / \Gamma(t),
\end{gathered}
$$

where $\vec{N}$ is the outward normal vector to $\Omega(t)$. Constitutive laws are needed for the percussions stresses and percussions $\Sigma$ and $\vec{R}$.

\subsection{The constitutive laws}

They result from a volume pseudopotential of dissipation $\Phi$ and a surface pseudopotential of dissipation $\Phi_{s}$. They are

$$
\Phi=\lambda D\left(\frac{\vec{U}^{+}+\vec{U}^{-}}{2}\right)^{2}, \Phi_{s}=\lambda_{s}\left(\frac{\vec{U}^{+}+\vec{U}^{-}}{2}\right)^{2}+I_{-}\left(U_{N}^{+}\right),
$$

where $U_{N}^{+}=\vec{U}^{+} \cdot \vec{N}$ is the normal velocity after the collision, $I_{-}$is the indicator function of the negative numbers $R^{-}$and, $\lambda$ and $\lambda_{s}$ are material parameters which can be determined by experiments [3]. The constitutive laws are

$$
\begin{gathered}
\Sigma=\frac{\partial \Phi}{\partial D\left(\left(\vec{U}^{+}+\vec{U}^{-}\right) / 2\right)}=\lambda D\left(\vec{U}^{+}+\vec{U}^{-}\right), \\
\vec{R}=\frac{\partial \Phi_{s}}{\partial\left(\left(\vec{U}^{+}+\vec{U}^{-}\right) / 2\right)} \in \lambda_{s}\left(\vec{U}^{+}+\vec{U}^{-}\right)+\partial I_{-}\left(U_{N}^{+}\right) \vec{N} .
\end{gathered}
$$

The quantity $\partial I_{-}\left(U_{N}^{+}\right) \vec{N}$ is the impenetrability percussion reaction. It is active only if the other actions are not sufficient for the solid and the plane not to interpenetrate, i.e., when the normal velocity after the collision is zero.

\subsection{The equations}

The equations giving the velocity field $\vec{U}^{+}$assuming the velocity field $\vec{U}^{-}$before the collision is known, result from the equation of motion (3.2), (3.3) and the constitutive laws (3.4), (3.5). They are

$$
\begin{gathered}
\rho[\vec{U}]=\lambda \operatorname{div} D\left(\vec{U}^{+}+\vec{U}^{-}\right), \text {in } \Omega(t), \\
\Sigma \vec{N}+\lambda_{s}\left(\vec{U}^{+}+\vec{U}^{-}\right)+\partial I_{-}\left(U_{N}^{+}\right) \vec{N} \ni 0, \text { in } \Gamma(t), \Sigma \vec{N}=\vec{T}_{e x t}, \text { in } \partial \Omega(t) / \Gamma(t) .
\end{gathered}
$$

It is an elliptic equation which can be easily solved

Theorem 3.1. If the domain $\Omega$ and the contact surface $\Gamma$ are smooth, $\vec{U}^{-} \in H^{1}(\Omega)$ and $\vec{T}_{\text {ext }} \in L^{2}(\Gamma)$, then there exist an unique velocity field after the collision $\vec{U}^{+} \in$ $H^{1}(\Omega)$. 


\section{Collisions with damage}

\subsection{Introduction}

Let consider a rock colliding a concrete protection wall. The very large interior

forces, the percussion stress $\Sigma$ and percussion $\vec{R}$ produce very fast microscopic motions which break the links which ensure the cohesion of the rock and of the concrete wall which damage. The damage quantity $\beta(\vec{x}, \tau)$ evolves rapidly in the collision. Thus we assume it is, as well as the macroscopic velocity $\vec{U}$, discontinuous at the collision time. There is damage $\beta^{-}(\vec{x}, t)$ before the collision and damage $\beta^{+}(\vec{x}, t)$ after the collision. For the sake of simplicity, we assume that the wall is a fixed, rigid and undamageable structure. Thus only the evolution of the rock is investigated.

\subsection{The equations of motion}

They result from the principle of virtual work. Let us recall that the work of the interior forces is a linear function of the strain rate $D(\vec{U})$ and of the velocity of damage $d \beta / d t$. Let us note that the strain rate $D(\vec{U})$ is a function of bounded variation with respect to time whereas the velocity $d \beta / d t$ is the derivative of a function of bounded variation with respect to time $(\beta(\vec{x}, \tau)$ is discontinuous at time $t)$. Thus it is wise to choose the work of the interior forces to be

$$
\begin{gathered}
\mathcal{T}_{i}(\vec{V}, b, \Omega)=-\int_{\Omega(t)} \Sigma: D\left(\frac{\vec{V}^{+}+\vec{V}^{-}}{2}\right) d \Omega-\int_{\Gamma(t)} \vec{R} \cdot\left(\frac{\vec{V}^{+}+\vec{V}^{-}}{2}\right) d \Gamma \\
-\int_{\Omega(t)}\{\hat{B}[b]+\hat{H} \cdot \overrightarrow{\operatorname{grad}}[b]\} d \Omega,
\end{gathered}
$$

where the virtual velocity field $\vec{V}(\vec{x}, \tau)$ and virtual damage velocity field $b(\vec{x}, \tau)$ are functions of bounded variation. The work of the acceleration forces is given by (3.1) because we neglect the density of the links compare to the density $\rho$. No exterior percussion is applied and no work is provided from the exterior to break the links. Thus the work of the exterior forces is zero. The equations of motion result from the principle of virtual work

$$
\forall \vec{V}, \forall b, \mathcal{T}_{a}(\vec{V}, \Omega)=\mathcal{T}_{i}(\vec{V}, b, \Omega) .
$$

They are

$$
\begin{gathered}
\rho[\vec{U}]=\operatorname{div} \Sigma, 0=\operatorname{div} \hat{H}-\hat{B}, \text { in } \Omega(t), \\
\Sigma \vec{N}=-\vec{R}, \text { in } \Gamma(t), \Sigma \vec{N}=0 \text {, in } \partial \Omega(t) / \Gamma(t), \hat{H} \cdot \vec{N}=0, \text { in } \partial \Omega(t),
\end{gathered}
$$

where $\vec{N}$ is the outward normal vector to $\Omega(t)$. 


\subsection{The constitutive laws}

The first and second laws of thermodynamics

$$
\begin{gathered}
\forall \mathcal{D}, \int_{\mathcal{D}}[e] d \Omega+\mathcal{T}_{a}(\vec{U}, \mathcal{D})=\int_{\mathcal{D}} Q^{e x t} d \Omega, \\
\forall \mathcal{D}, 0 \geq \int_{\mathcal{D}} \frac{Q^{e x t}}{T} d \Omega
\end{gathered}
$$

where $T$ is the temperature assumed to be constant, $e$ is the internal energy and, $Q^{e x t}$ is the heat impulse provided by the exterior in the collision. The principle of virtual work (4.1) with the actual velocities and the first law (4.4) give

$$
\forall \mathcal{D}, \int_{\mathcal{D}}[e] d \Omega=-\mathcal{T}_{i}(\vec{U}, \mathcal{D})+\int_{\mathcal{D}} Q^{e x t} d \Omega .
$$

With the second law we have

$$
\forall \mathcal{D}, \int_{\mathcal{D}}[\Psi] d \Omega \leq-\mathcal{T}_{i}(\vec{U}, \mathcal{D}),
$$

where $\Psi$ is the free energy, (in the present situation, $e=\Psi$ ). It results

$$
\begin{gathered}
{[\Psi] \leq \Sigma: D\left(\frac{\vec{U}^{+}+\vec{U}^{-}}{2}\right)+\hat{B}[\beta]+\hat{H} \cdot \overrightarrow{\operatorname{grad}}[\beta], \text { in } \Omega,} \\
0 \leq \vec{R} \cdot \frac{\vec{U}^{+}+\vec{U}^{-}}{2}, \text { in } \Gamma .
\end{gathered}
$$

We denote $\underline{\beta}$ the average value of $\beta$

$$
\underline{\beta}=\frac{\beta^{+}+\beta^{-}}{2}=\beta^{-}+\frac{[\beta]}{2} .
$$

The constitutive laws are defined with three functions: the free energy, $\Psi$, the volume pseudopotential of dissipation, $\Phi$ and the surface pseudopotential of dissipation $\Phi_{s}$

$$
\begin{gathered}
\Psi(\epsilon, \beta, \overrightarrow{\operatorname{grad}} \beta)=w(1-\beta)+\frac{\beta}{2} \epsilon K \epsilon+\frac{k}{2}(\overrightarrow{\operatorname{grad}} \beta)^{2}+I(\beta), \\
\Phi\left(D\left(\frac{\vec{U}^{+}+\vec{U}^{-}}{2}\right),[\beta], \operatorname{grad}[\beta]\right)=\frac{c}{2}[\beta]^{2}+\mu\left(\beta^{-}+\frac{[\beta]}{2}\right)\left\|D\left(\vec{U}^{+}+\vec{U}^{-}\right)\right\| \\
+\lambda\left(D\left(\frac{\vec{U}^{+}+\vec{U}^{-}}{2}\right)\right)^{2}+I_{-}([\beta]), \\
\Phi_{s}\left(\frac{\vec{U}^{+}+\vec{U}^{-}}{2}\right)=\lambda_{s}\left(\frac{\vec{U}^{+}+\vec{U}^{-}}{2}\right)^{2}+I_{-}\left(U_{N}^{+}\right),
\end{gathered}
$$

where $U_{N}^{+}=\vec{U}^{+} \cdot \vec{N}$ is the normal velocity and $I, I_{-}$are the indicator functions of the segment $[0,1]$ and of the set of the negative numbers $R^{-}$(see appendix). The 
quantity $w$ is the cohesion energy of the material, $K$ is the elasticity tensor, $\epsilon$ the deformation which does not change in the collision, and $k$ is the damage coefficient which quantifies the influence of the damage at a point onto the damage of its neighbourhood. The parameters $c$ and, $\lambda, \mu, \lambda_{s}$ caracterise the microscopic and macroscopic dissipations in collisions. The norm of tensor $E$ is defined by

$$
\|E\|=\sqrt{E_{i j} E_{i j}}=\sqrt{E^{2}} .
$$

The indicator functions take care of the internal constraints

$$
\beta \in[0,1],[\beta] \leq 0, U_{N}^{+} \leq 0 .
$$

The first internal constraint results from the relationship (1.1) [4], [8]. The second internal constraint means that the solid does not mend in the collision; the third is the impenetrability condition of the two solids. The surface dissipative function $\Phi_{s}\left(\frac{\vec{U}^{+}+\vec{U}^{-}}{2}\right)$ is a pseudopotential of dissipation The volume dissipative function $\Phi\left(D\left(\frac{\vec{U}^{+}+\vec{U}^{-}}{2}\right),[\beta], \operatorname{grad}[\beta]\right)$ is also a pseudopotential of dissipation provided $\mu^{2} \leq$ $2 c \lambda$. This relationship is assumed in the following.

We can verify that the free energy is a convex function of $(\beta, \overrightarrow{\operatorname{grad}} \beta)$ and prove a general result

Theorem 4.1. If the free energy $\Psi$ is a convex function of $(\beta, \overrightarrow{g r a d} \beta)$, we have

$$
\begin{gathered}
\forall\left(\hat{B}^{n d}, \hat{H}^{n d}\right) \in \partial \Psi\left(\beta^{+}, \overrightarrow{\operatorname{grad}} \beta^{+}\right), \\
{[\Psi] \leq \hat{B}^{n d}[\beta]+\hat{H}^{n d} \cdot \overrightarrow{\operatorname{grad}}[\beta],}
\end{gathered}
$$

where the subdifferential of $\Psi$ is computed with respect to the last two variables $\beta, \overrightarrow{\operatorname{grad}} \beta$.

Proof. Let

$$
\left(\hat{B}^{n d}, \hat{H}^{n d}\right) \in \partial \Psi\left(\beta^{+}, \overrightarrow{\operatorname{grad}} \beta^{+}\right),
$$

where the deformation $\epsilon$ is kept constant. Thus

$$
\begin{aligned}
\hat{B}^{n d}\left(\beta^{-}-\beta^{+}\right)+\hat{H}^{n d} \cdot\left(\overrightarrow{\operatorname{grad}} \beta^{-}-\overrightarrow{\operatorname{grad}} \beta^{+}\right) & +\Psi\left(\epsilon, \beta^{+}, \overrightarrow{\operatorname{grad}} \beta^{+}\right) \\
& \leq \Psi\left(\epsilon, \beta^{-}, \overrightarrow{\operatorname{grad}} \beta^{-}\right) .
\end{aligned}
$$

This relationship gives

$$
\begin{aligned}
{[\Psi] } & =\Psi\left(\epsilon, \beta^{+}, \overrightarrow{\operatorname{grad}} \beta^{+}\right)-\Psi\left(\epsilon, \beta^{-}, \overrightarrow{\operatorname{grad}} \beta^{-}\right) \\
& \leq-\hat{B}^{n d}\left(\beta^{-}-\beta^{+}\right)-\hat{H}^{n d} \cdot\left(\overrightarrow{\operatorname{grad}} \beta^{-}-\overrightarrow{\operatorname{grad}} \beta^{+}\right) \\
& =\hat{B}^{n d}[\beta]+\hat{H}^{n d} \cdot \overrightarrow{\operatorname{grad}}[\beta] .
\end{aligned}
$$

Thus

$$
[\Psi] \leq \hat{B}^{n d}[\beta]+\hat{H}^{n d} \cdot \overrightarrow{\operatorname{grad}}[\beta]
$$




\subsubsection{The constitutive laws in $\Omega$ and in $\Gamma$}

We choose the constitutive laws

$$
\begin{gathered}
(\Sigma,(\hat{B}, \hat{H})) \in\left\{0, \partial \Psi\left(\beta^{+}, \overrightarrow{\operatorname{grad}} \beta^{+}\right)\right\}+\partial \Phi\left(D\left(\frac{\vec{U}^{+}+\vec{U}^{-}}{2}\right),[\beta], \operatorname{grad}[\beta]\right), \\
\vec{R} \in \partial \Phi_{s}\left(\frac{\vec{U}^{+}+\vec{U}^{-}}{2}\right)
\end{gathered}
$$

which give

$$
\begin{gathered}
\Sigma=\Sigma^{c}+\lambda D\left(\vec{U}^{+}+\vec{U}^{-}\right), \\
\Sigma^{c} \in \partial J\left(D\left(\vec{U}^{+}+\vec{U}^{-}\right)\right),
\end{gathered}
$$

with

$$
\partial J(D(\vec{V}))=\left\{E \mid E=2 \mu \underline{\beta} \frac{D(\vec{V})}{\|D(\vec{V})\|} \text { if } D(\vec{V}) \neq 0 ;\|E\| \leq 2 \mu \underline{\beta} \text { if } D(\vec{V})=0\right\},
$$

and

$$
\begin{gathered}
\hat{B} \in-w+\frac{1}{2} \epsilon K \epsilon+\partial I\left(\beta^{+}\right)+c[\beta]+\frac{\mu}{2}\left\|D\left(\vec{U}^{+}+\vec{U}^{-}\right)\right\|+\partial I_{-}([\beta]), \\
\hat{H}=k \overrightarrow{g r a d} \beta^{+}, \\
\vec{R} \in \lambda_{s}\left(\vec{U}^{+}+\vec{U}^{-}\right)+\partial I_{-}\left(U_{N}^{+}\right) \vec{N} .
\end{gathered}
$$

There is no dissipation with respect to $\overrightarrow{\operatorname{grad}}[\beta]$ thus $\hat{H}=\hat{H}^{\text {nd }}$. We can prove that the constitutive laws (4.9)-(4.14) are such that the second law (4.5) and (4.6) is satisfied

Theorem 4.2. If the constitutive laws (4.9)-(4.14) are satisfied, then the second law of thermodynamics is satisfied and the internal constraints (4.7) are satisfied.

Proof. From the constitutive law (4.9) and the properties of pseudopotentials we get

$$
0 \leq \Sigma: D\left(\frac{\vec{U}^{+}+\vec{U}^{-}}{2}\right)+\left(\hat{B}-\hat{B}^{n d}\right)[\beta]+\left(\hat{H}-\hat{H}^{n d}\right) \cdot \overrightarrow{\operatorname{grad}}[\beta] .
$$

and from relationship (4.8)

$$
[\Psi]-\hat{B}^{n d}[\beta]-\hat{H}^{n d} \cdot \overrightarrow{\operatorname{grad}}[\beta] \leq 0 .
$$

Thus

$$
\begin{gathered}
{[\Psi]-\hat{B}^{n d}[\beta]-\hat{H}^{n d} \cdot \overrightarrow{\operatorname{grad}}[\beta] \leq 0} \\
\leq \Sigma: D\left(\frac{\vec{U}^{+}+\vec{U}^{-}}{2}\right)+\left(\hat{B}-\hat{B}^{n d}\right)[\beta]+\left(\hat{H}-\hat{H}^{n d}\right) \cdot \overrightarrow{\operatorname{grad}}[\beta] .
\end{gathered}
$$

which gives the relationship (4.5). The relationship (4.6) is satisfied due to the properties of pseudopotential $\Phi_{s}$. The internal constraints $\beta \in[0,1],[\beta] \leq 0$ and $U_{N}^{+} \leq 0$ are satisfied because the various subdifferential sets are not empty (see appendix). 


\subsection{An example : a rock colliding a rigid surface}

When a rock collides a rigid surface no exterior percussion is applied. The equations of motion (4.2)-(4.3) and the constitutive laws (4.9)-(4.14) give the equations to compute the velocity and damage of the rock after the collision $\vec{U}^{+}$and $\beta^{+}$, depending on the velocity and damage before the collision $\vec{U}^{-}$and $\beta^{-}$

$$
\begin{gathered}
\rho[\vec{U}]=\operatorname{div}\left(\Sigma^{c}+\lambda D\left(\vec{U}^{+}+\vec{U}^{-}\right)\right), \text {in } \Omega, \\
c[\beta]-k \Delta \beta^{+}+\partial I\left(\beta^{+}\right)+\partial I_{-}([\beta]) \ni w-\frac{1}{2} \epsilon K \epsilon-\frac{\mu}{2}\left\|D\left(\vec{U}^{+}+\vec{U}^{-}\right)\right\|, \text {in } \Omega \\
\Sigma \vec{N}+\lambda_{s}\left(\vec{U}^{+}+\vec{U}^{-}\right)+\partial I_{-}\left(U_{N}^{+}\right) \vec{N} \ni 0 \text { in } \Gamma, \Sigma \vec{N}=0, \text { in } \partial \Omega / \Gamma, \\
\frac{\partial \beta^{+}}{\partial N}=0, \text { in } \partial \Omega .
\end{gathered}
$$

We assume that the rock is undamaged before the collision and that its velocity is a rigid body velocity

$$
\beta^{-}=1, D\left(\vec{U}^{-}\right)=0 \text {. }
$$

The equations to find the damage $\beta^{+}$and, the velocity $\vec{U}^{+}$after the collisions are

$$
\begin{gathered}
\rho \vec{U}^{+}-\operatorname{div}\left(\Sigma^{c}+\lambda D\left(\vec{U}^{+}\right)\right)=\rho \vec{U}^{-} \\
c \beta^{+}-k \Delta \beta^{+}+\partial I\left(\beta^{+}\right)+\partial I_{-}\left(\beta^{+}-1\right) \ni w+c-\frac{1}{2} \epsilon K \epsilon-\frac{\mu}{2}\left\|D\left(\vec{U}^{+}\right)\right\|, \text {in } \Omega \\
\Sigma \vec{N}+\lambda_{s}\left(\vec{U}^{+}+\vec{U}^{-}\right)+\partial I_{+}\left(U_{N}^{+}\right) \vec{N} \ni 0 \text { in } \Gamma, \Sigma \vec{N}=0 \text { in } \partial \Omega / \Gamma \\
\frac{\partial \beta^{+}}{\partial N}=0, \text { in } \partial \Omega
\end{gathered}
$$

Remark 2. We have $\partial I\left(\beta^{+}\right)+\partial I_{-}\left(\beta^{+}-1\right)=\partial I\left(\beta^{+}\right)$, thus equation (4.20) becomes

$$
c \beta^{+}-k \Delta \beta^{+}+\partial I\left(\beta^{+}\right) \ni w+c-\frac{1}{2} \epsilon K \epsilon-\frac{\mu}{2}\left\|D\left(\vec{U}^{+}\right)\right\|, \text {in } \Omega .
$$

\subsection{An existence theorem}

The set of equations (4.19), (4.21), (4.22) and (4.23) has a variational formulation. Let $C$ be the convex set

$$
C=\left\{(\vec{V}, \delta) \mid V_{N} \leq 0 \text { in } \Gamma ; \delta \in[0,1]\right\} .
$$

We have

$$
\left(\vec{U}^{+}, \beta^{+}\right) \in C
$$


and by classical computations

$$
\begin{gathered}
\forall(\vec{V}, \delta) \in C \\
\int_{\Omega} \rho \vec{U}^{+} \cdot\left(\vec{V}-\vec{U}^{+}\right) d \Omega+\int_{\Omega} c \beta^{+}\left(\delta-\beta^{+}\right) d \Omega+\int_{\Omega} k \overrightarrow{\operatorname{grad}} \beta^{+} \cdot \overrightarrow{\operatorname{grad}}\left(\delta-\beta^{+}\right) d \Omega \\
\quad+\int_{\Omega} \lambda D\left(\vec{U}^{+}\right):\left(D(\vec{V})-D\left(\vec{U}^{+}\right)\right) d \Omega \\
+\mu \int_{\Omega}\left(\frac{1+\delta}{2}\right)\|D(\vec{V})\| d \Omega-\mu \int_{\Omega}\left(\frac{1+\beta^{+}}{2}\right)\left\|D\left(\vec{U}^{+}\right)\right\| d \Omega \\
+\lambda_{s} \int_{\Gamma} \vec{U}^{+} \cdot\left(\vec{V}-\vec{U}^{+}\right) d \Gamma \geq \int_{\Omega} \rho \vec{U}^{-} \cdot\left(\vec{V}-\vec{U}^{+}\right) d \Omega \\
-\lambda_{s} \int_{\Gamma} \vec{U}^{-} \cdot\left(\vec{V}-\vec{U}^{+}\right) d \Gamma+\int_{\Omega}\left(w+c-\frac{1}{2} \epsilon K \epsilon\right)\left(\delta-\beta^{+}\right) d \Omega
\end{gathered}
$$

It can be proved that this variational inequality has a unique solution

Theorem 4.3. If the domain $\Omega$ and the nonzero measure contact surface $\Gamma$ are smooth, if the velocity before the collision $\vec{U}^{-}$is a rigid body velocity, if the elastic energy $\frac{1}{2} \epsilon K \epsilon \in L^{1}(\Omega)$, then the variational inequality (4.24), (4.25) has a unique solution $\left(\vec{U}^{+}, \beta^{+}\right) \in\left(H^{1}(\Omega)\right)^{3} \times H^{1}(\Omega)$.

\subsection{Properties of the solution}

The equation (4.19) shows that the strain rate $D\left(\vec{U}^{+}\right)$increases with $\vec{U}^{-}$. Let assume the damage $\beta^{+}$to be homogeneous after the collision $\left(\Delta \beta^{+}=0\right)$. Then the equation (4.23) gives, assuming the rock is not deformed when colliding $(\epsilon=0)$

$$
c \beta^{+}+\partial I\left(\beta^{+}\right) \ni w+c-\frac{\mu}{2}\left\|D\left(\vec{U}^{+}\right)\right\| .
$$

The solution of this equation is

$$
\begin{gathered}
\beta^{+}=1, \text { if } \frac{1}{c}\left(w+c-\frac{\mu}{2}\left\|D\left(\vec{U}^{+}\right)\right\|\right) \geq 1, \\
\beta^{+}=\frac{1}{c}\left(w+c-\frac{\mu}{2}\left\|D\left(\vec{U}^{+}\right)\right\|\right) \text {, if } 0 \leq \frac{1}{c}\left(w+c-\frac{\mu}{2}\left\|D\left(\vec{U}^{+}\right)\right\|\right) \leq 1, \\
\beta^{+}=0, \text { if } \frac{1}{c}\left(w+c-\frac{\mu}{2}\left\|D\left(\vec{U}^{+}\right)\right\|\right) \leq 0 .
\end{gathered}
$$

This result proves that if the incoming velocity is low the collision produces no damage (case (4.26)). If it is large, it produces damage (cases (4.27) and (4.28)). The larger is the velocity, the larger is the damage. Fracture occurs when $\beta^{+}=0$ (case (4.28)).

It is possible to prove that if the parameter $\lambda \rightarrow \infty$, the equations become the rigid body collision equations [4] and no damage occurs because $D\left(\vec{U}^{+}\right)$becomes zero. 


\section{Appendix. Convex functions}

Let us consider the set $R \cup\{+\infty\}$ where the addition and the multiplication by strictly positive numbers are completed by the rules $+\infty+b=+\infty$ and $a(+\infty)=$ $+\infty(a>0)$. A function from $R^{n}$ into $R \cup\{+\infty\}$ is convex iff

$$
\left.\forall x, y \in \mathbf{R}^{n}, \forall \theta \in\right] 0,1[, f(\theta x+(1-\theta) y) \leq \theta f(x)+(1-\theta) f(y) .
$$

A convex function in not always differentiable but a generalised derivative may be defined: an element $z \in R^{n}$ is a subgradient of the convex function $f$ at point $x$ iff

$$
\forall y \in \mathbf{R}^{n}, f(y) \geq f(x)+(y-x) \cdot z .
$$

The set of the sub-gradients is denoted $\partial f(x)$, the subdifferential set. The indicator function, $I_{-}$, of the set of the negative numbers $R^{-}$is defined by $I_{-}(x)=0$ if $x \leq 0$ and $I(x)=+\infty$ if $x>0$. It is a convex function. Its subdifferential set is shown in figure 1 . The indicator function $I$ of the segment $[0,1]$ is defined by $I(x)=0$ if $x \in[0,1]$ and $I(x)=+\infty$ if $x \notin[0,1]$. It is a convex function. Its subdifferential set is shown in figure 1 .
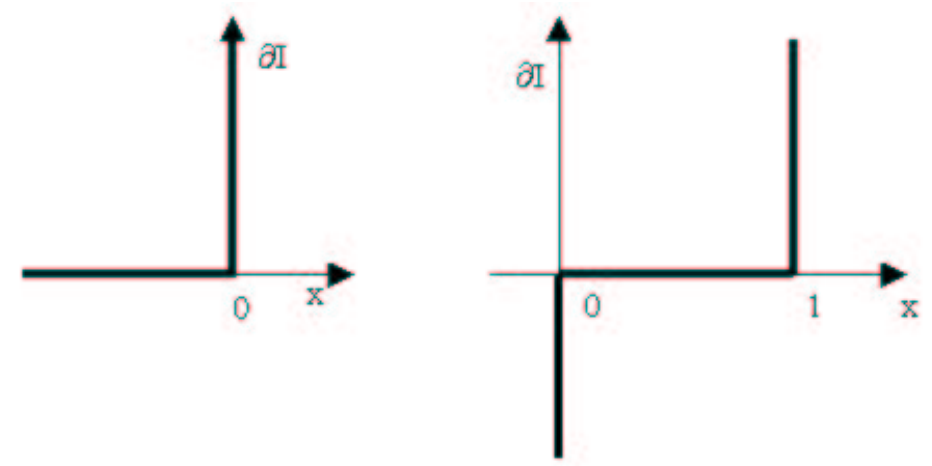

Figure 1: The subdifferential set $\partial I_{-}$of the indicator function of the negative numbers $R^{-}: \partial I_{-}(0)=R^{+}, \partial I_{-}(x)=\{0\}$ for $x<0$ and $\partial I_{-}(x)=\emptyset$ for $x>0$. The subdifferential set $\partial I$ of the indicator function $I$ of the segment $[0,1]$.

The following theorem is useful for reading the constitutive laws

Theorem 5.1. Let a convex function $f \neq+\infty$. If this function is subdifferentiable at point $x$, i.e. if $\partial f(x) \neq \emptyset$, it is finite at this point: $f(x)<+\infty$.

This theorem applies in the numerous constitutive laws such as $B \in \partial I(\beta)$ : because $\partial I(\beta)$ is not empty (it contains $B$ ), it results from theorem 5.1 that $I(\beta)<+\infty$ which implies $I(\beta)=0$ and $0 \leq \beta \leq 1$, see the figure 1 . Thus the constitutive law $B \in \partial I(\beta)$ implies that the constraint $0 \leq \beta \leq 1$ on the quantity $\beta$ is satisfied. The constitutive law has two meanings: first it implies that the constraint is satisfied, second it gives the value of the reaction to the constraint. 


\section{References}

[1] C. Cholet, "Chocs de Solides Rigides", Thèse, Université Pierre et Marie Curie, Paris, 1998.

[2] H. da Costa Mattos, M. Frémond, E.N. Mamiya, A simple model of the mechanical behaviour of ceramic like material, Int. J. Solids Struct., 29, No. 24 (1992), 3185-3200.

[3] E. Dimnet, "Collisions de Solides Déformables", Thèse, Ecole nationale des Ponts et Chaussées, Paris, 2002.

[4] M. Frémond, "Non-smooth Thermomechanics", Springer-Verlag, Heidelberg, 2001.

[5] M. Frémond, 2002, Damage theory. A macroscopic motion vanishes but its effects remain, Computational and Applied Mathematics, 21, No. 2 (2002), to appear.

[6] M. Frémond, R. Gormaz, J. San martin, Collision of a solid with an uncompressible fluid, to appear.

[7] J.J. Moreau, Sur les lois de frottement, de viscosité et de plasticité, C. R. Acad. Sci., Paris, 271 (1970), 608-611.

[8] B. Nedjar, 1995, "Mécanique de L'endommagement. Théorie du Premier Gradient et Application au Béton", Thèse, Ecole nationale des Ponts et Chaussées, Paris, 1995. 